\title{
Which of two individuals do you treat when only their ages are different and you can't treat both?
}

\author{
P A Lewis and M Charny University of Wales College of Medicine and Bath District Health Authority, \\ respectively
}

\section{Authors' abstract}

A relative value of life dependent on age has been produced from a survey of 721 randomly selected individuals together with other observations of professional practice. The results are presented in diagrammatic form. If two identical people, except for age, present for medical treatment for a life-threatening condition and only one can be treated then the diagram indicates what the choice should be.

Many people react with distaste at an attempt to ascribe a value to a particular human life. When faced with the hypothetical question of how much time, effort and resource should be invested in trying to save a particular life there is a widely held view that no expense should be spared. Yet there are analyses which demonstrate that society behaves as if there is a finite and not very large value of a life and therefore the amount of resource that maybe invested to try to preserve that life. This is inevitable since the demands made upon society exceed its finite resources. There are conflicting claims between sectors - for example health, housing, transport etc, but even within health there are more claims for treatment than can be provided, making selection inescapable. The study of the 'value' of a human life has more to do with the need to evaluate different mortality/morbidity-reducing strategies than with any fundamental desire to evaluate the worth of a life. If, as is the case, resources available for the reduction of the risk of mortality and morbidity are finite then a choice exists between different strategies, affecting differing numbers of different people in different ways. Some common denominator of costs and benefit is needed if the greatest benefit is to be obtained from the use of those resources. A monetary value of the 'worth' of a life is chosen only because costs are usually expressed in this way. Thus valuing life is a means to an end and the unease which many people feel at the concept of placing a value on a life stems from a misconception that it represents an end in itself.

\section{Key words}

Valuation of life; age preference; treatment decisions.
The value of a life is required if decision-makers are to evaluate the benefits of the risk reductions that are being purchased using different strategies or combinations of strategies. While attention tends to be focussed on the fact that such decisions may result in certain individuals dying when they might otherwise have lived, it is clear that in the future more individuals will be alive who might otherwise be dead if the greatest good for the greatest number is pursued. Society is accepting more explicitly that such choices need to be made in the name of 'greater good' if more people are to benefit and fewer suffer as a result of health service provision. There is much debate on the practical interpretation of the concept of greater good and controversy still exists on reasonable ways of applying it, for example (1). At its simplest there is often a wide discrepancy between the optimum solution to a problem in terms of society as a whole and from the perspective of an individual member of that society but this follows inevitably from the fact that we all value our own welfare and that of the individuals close to us much more highly than those not known to us. For a much deeper presentation of these issues see $(2,3)$ and $(4)$.

For the doctor in a clinic these 'abstract' choices between groups of people are not relevant. The doctor's principal concern is to decide whether the risks of a particular treatment outweigh the benefits for the individual patient under consideration. In an individual doctor-patient contact, the doctor is not concerned with the effect that his decision will have on other patients in the future, or those who are being seen by other doctors. For the planner, however, consideration has to be given to the circumstances in which two patients will benefit from treatment when there are only sufficient resources to treat one of them. At present these decisions are taken by large numbers of physicians working in isolation, and the result is a haphazard aggregation of individual decisions. Effectively society is represented in the decisionmaking process by samples of one, which we suggest is unrepresentative and thus inescapably unsatisfactory. Society should not ask doctors to bear the burden of this decision-making, not only because it is inefficient but also because the choices (as distinct from the implementation of these choices) should have a social 
rather than professional base. This suggests that the question of whether society at large holds a view regarding who should be treated under such circumstances is important. If so, can such views be ascertained and embodied in decision-making principles for use when such choices need to be made?

In order to test the hypothesis that 'society' would be prepared to choose under such circumstances, some questions on such choices were included in the recent Cardiff Health Survey $(5,6)$. Among fifteen such scenarios three questions focused on choosing between two people alike in all respects other than their ages. The part of the questionnaire used for these questions is given in Table 1. This shows the instructions to the interviewer as well as the information given to the interviewee. A total of 721 individuals chosen at random from the electoral register for Cardiff City were asked to make these choices. The resulting frequencies are given in Table 2 . For the first two choices, those that expressed an opinion opted for the younger in the ratio of $84: 1$ and $14: 1$ respectively. However, when choosing between the two-year-old and the eight-year-old, the choice was for the older by a ratio of $5: 3$.

As part of the validation procedure for the questionnaires, in the pilot stage of the study, a random sample of 54 drawn from the same base population was asked to complete the questionnaire and were then subsequently interviewed on their responses, the interviews being taped. In summary the reasons given to favour the eight-year-old are to protect the investments that have already been made, and the

\section{Table 1}

SAY: 'In the next section, I want to tell you about some imaginary situations. After each one I'm going to ask you to make a choice. Assume that the people are the same in every respect except the one I'll tell you about'.

SAY: 'Imagine two people, both with leukaemia, one aged 5 and the other aged 70'.

SAY: 'If only one of these people can be treated, which one do you think it should be?' WHEN THE RESPONDENT HAS ANSWERED, ASK: "Did you find that making this choice was very difficult, quite difficult, quite easy or very easy'.

Score as below according to the respondent's reply.

$\begin{array}{ccccc} & \begin{array}{c}\text { Very } \\ \text { Difficult }\end{array} & \begin{array}{c}\text { Quite } \\ \text { Difficult }\end{array} & \begin{array}{c}\text { Quite } \\ \text { Easy }\end{array} & \begin{array}{c}\text { Very } \\ \text { Easy }\end{array} \\ \text { Person A } & 4 & 3 & 2 & 1 \\ \text { Person B } & 6 & 7 & 8 & 9\end{array}$

IF THE RESPONDENT SAYS THAT HE/SHE CANNOT MAKE A CHOICE, ENCOURAGE

THEM ONCE TO TRY TO DO SO. IF NO CHOICE IS MADE, SCORE 5. IF A CHOICE IS MADE ASK SUPPLEMENTARY QUESTION AND SCORE ACCORDING TO THE SCHEME FOR PERSON A AND PERSON B.

\section{Table 2}

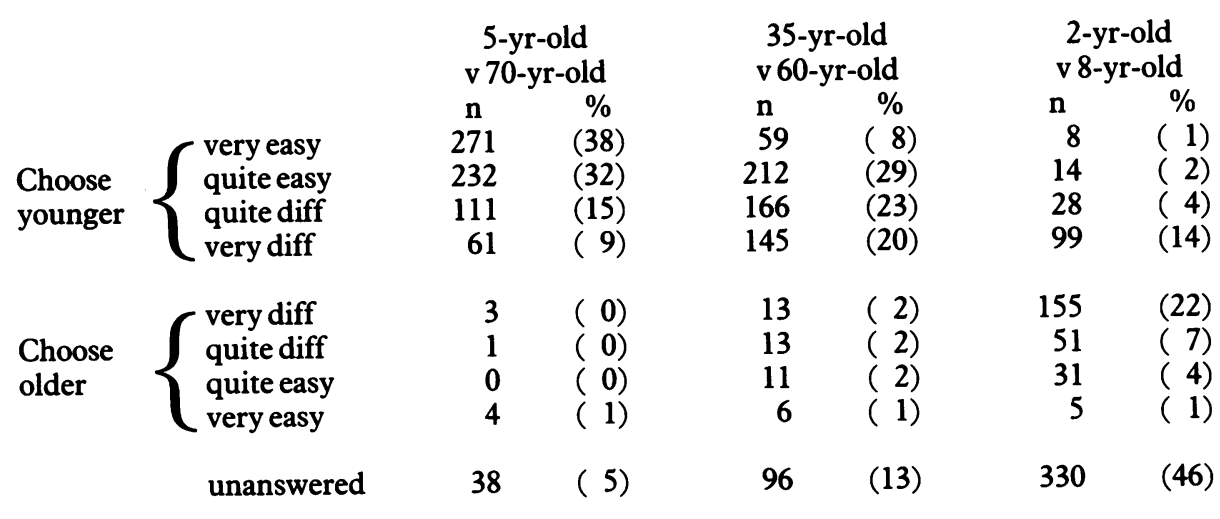


additional suffering and anguish on the part of the eight-year-old because eight-year-olds better understand their circumstances. While we have no information from the study sample on the arguments used by each of the 242 individuals who voted for the older child, the rejection of the notion that youth necessarily takes precedence reveals a sophistication amongst the responders which may be surprising to some.

In further exploring and analysing the arguments used to favour the older child we must recognise that while an eight-year-old was chosen in preference to a two-year-old there will be some older age than eight (as yet unspecified) at which the two-year-old will be chosen, in order to maintain a consistency with the other two age-related choices.

In the survey no one articulated the 'rights' of the younger child, but they seem straightforward. The younger child will be losing more of his/her life than the older and therefore loses more in this respect. The challenge, which is in part attempted later, is to decide which point marks the boundary between choosing the younger and choosing the older.

If a choice can always be made between two individuals who are identical in all respects except age, then it should be possible to map that choice. This process can draw on a variety of observations to supplement the three already found and reported above in the Cardiff Health Survey. The extra observations are set out below.

i) The treatment of infertility should not be at the expense of the treatment of the already conceived. This idea was prompted by Smith (7). That is, that the conceived should get preference over the not yet conceived. The reasonableness of this must be judged against the requirement that in all other respects the two individuals should be identical. Thus circumstances might arise whereby the other factors in the cases might lead to a reversal of this decision. This is analogous to the decision many women take to have an abortion.

ii) A fetus that has passed the stage whereby it is capable of normal growth and survival outside the womb should be treated in preference to one which has not.

iii) A woman who is pregnant should be treated in preference to the unborn child when there is a choice beween saving her or her child. If a mother cannot be expected to sacrifice her life for that of her fetus then we cannot expect an equivalent third party to sacrifice his/her life for someone else's unborn child. Because we impose the conditions that all people are the same except for age, the same criteria that have been applied to the mother must be applied to the third parties. This is not to deny that many individuals would choose to sacrifice themselves, merely that it would be illogical for society to require that sacrifice. We can however show that there is an age-related change in women. Women reach menopause by about 45 years of age. Thus the rule about choosing between a mother and her fetus can only be safely generalised to saving the adult until the adult reaches (say) 45 . This is not to say that an empirical study would not reveal an older age.

iv) A person who is already alive should be treated in preference to one who is not yet conceived. The consequence of this should be that the sub-fertile cannot use the 'rights' of their as yet unconceived children to improve their case for treatment.

v) A living person who can be treated successfully should be treated in preference to a not yet viable fetus.

vi) Further we may assume that when two individuals are alike in all respects including the same age then it should be impossible to choose between them. This is the situation in which we are indifferent to the choice made.

The consequences of these extra observations have been summarised, in terms of the implied solution to the problem, in Fig 1. Those parts of the solution boundary relating to the observations above have been labelled with the appropriate matching Roman numeral.

The solution has been primarily defined by these extra observations as indicated. While there is certain to be debate as to the exact location of particular points there seems to be a basis for achieving a consensus for most of the boundary. The difficult part of the boundary is that region marked (vii), because the only evidence we have of its location is one of its (imprecise) ends together with data concerning choice derived from the Cardiff Health Survey. The boundary between the two solutions is the point where the conflict between the potential years of life lost, the investments that have been made and the anguish of knowing what is happening pull in opposite directions. It can be argued that there comes a point in life where society ceases 'investing' in individuals. Suppose that this point is when education and vocational training has ceased and that it occurs at 23 years of age. Thus choosing between two people aged 23 and say 24 , the 23-year-old would be chosen as he/she had the same investment, the same anguish, but more years to lose. On the other hand, when choosing two people aged 23 and, say 22, the 23-year-old might be chosen due to the extra investment that has been made; the anguish would be comparable and the extra year of life lost by the younger of less value than the extra year of investment in the older. To ensure that these age pairs are the correct side of the boundary, the boundary must leave the equal age line and move down and to the right. Eventually this part of the boundary joins up with that part due to (iii) above. It has been drawn as concave to the older group in order to reflect the extra years lost by the younger groups. The curve must be continuous but it is not self-evident that a consensus view would choose a 23-year-old in favour of say a 14year-old, and the curve may therefore bend back upon itself.

Empirical research might eventually show that the boundary departs the equal age line moving down and to the left. The authors feel that this would be 
Fig 1: Map to show which of two patients to treat when their only difference is their ages.

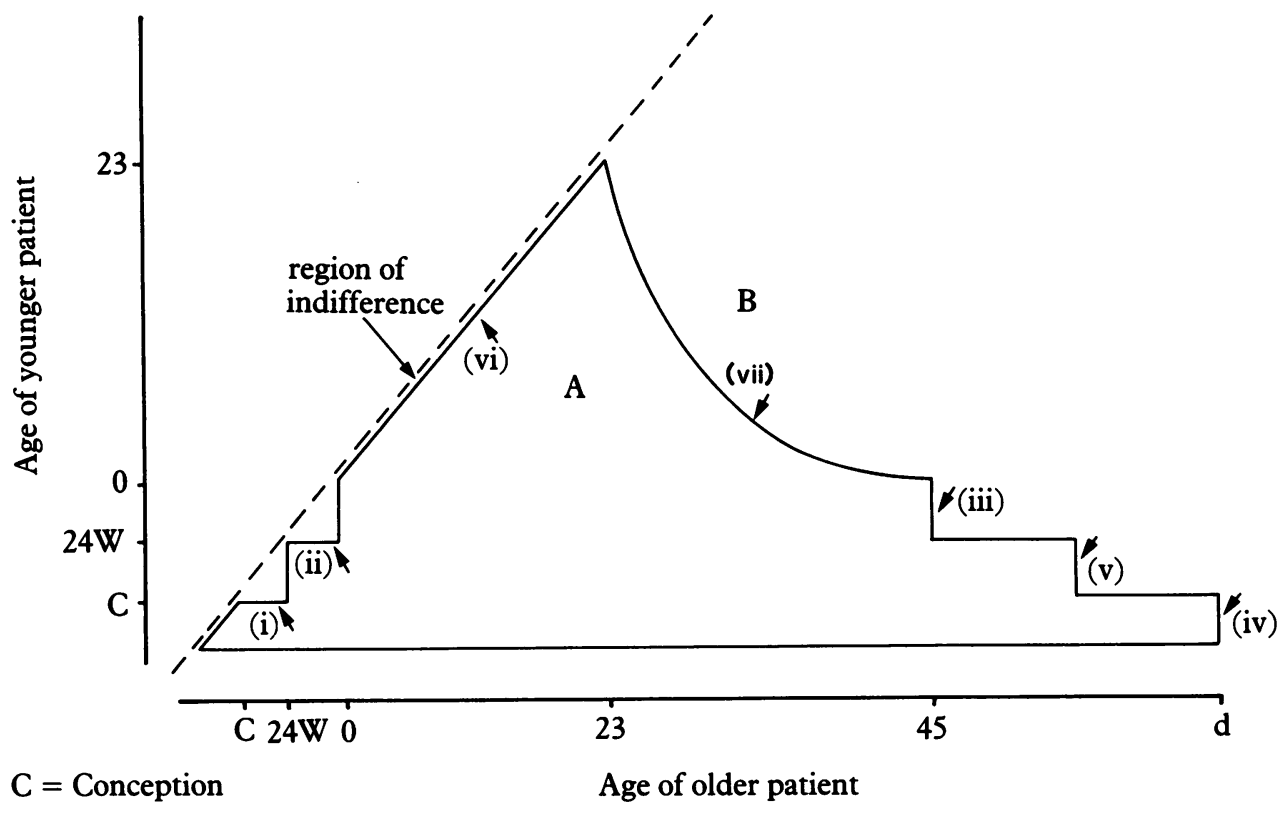

$24 \mathrm{~W}=24$ weeks - limit of viable life.

\section{Note}

To use this figure locate the point representing the age of the older and younger patients. This should lie in the lower triangular area. If the point lies within the perimeter (A) then the older patient should be treated. If the point lies outside this area (B) then the younger patient should be treated. A point on the perimeter indicates that you cannot use age as a criterion for choosing between the individuals. inconsistent with their observations. In the region of 23 years the marginal change in anguish will be zero. For the curve to move down and to the left implies that the value of a year lost is greater than the value of the investment made in that year. However, the consequence of choosing the eight-year-old in favour of the two-year-old is to suggest otherwise. It is being assumed here that the same principle applies equally all over the solution space. The apparent sophistication of the respondents to this study has been noted above and it would be unwise not to expect equal sophistication elsewhere, but not necessarily the same principle.

The observations from the Cardiff Health Survey which show that there is a strong body of opinion that there are cases where an older living individual should be saved in preference to an otherwise identical younger living individual, focuses attention on the problem that doctors face on a day-to-day basis when dealing with finite resources. The methodology used in the Cardiff Health Survey could be used together with this analysis to identify more closely the boundary between the choice for the older and the choice for the younger, especially as there is now enough data to give an approximate solution and guide the search. The benefit of achieving an accurate map would be that decision-making in health services could begin to reflect the values of the members of society as a whole, who pay for the health services offered. In other words, it is an attempt to bring true democracy into health service decision-making. This paper has concentrated on choices based on age alone. The Cardiff Health Survey has explored choices based on other criteria (sex, social class, marital status etc) and it is possible to $\stackrel{ }{工}$ conceive of producing similar maps to that of Fig 1 for $\frac{D}{0}$ a number of other variables which could eventually be used in conjunction with one another to generate a $\widetilde{N}$ multi-dimensional solution space (rather than the two- $N$ dimensional solution space which results from examining a single variable).

\section{Footnote}

If it has been decided previously that resources be made available to treat a specified number of patients? with a given condition, the random variation in patients presenting will mean that on some days $\stackrel{\mathrm{O}}{\mathrm{O}}$ patients will be treated with a lower 'value of life' than $\frac{?}{\square}$ that anticipated and on others patients who would $\stackrel{\mathbb{Q}}{ }$ otherwise have qualified for treatment will be preempted by even more valuable lives. This is because 8 many of the health sector resources are temporal. You cannot save surgeon-time or equipment-time from one week to the next on a regular basis. If a machine is not $\stackrel{?}{\rightleftharpoons}$ 
used one day you cannot use it for 48 hours the next day.

P A Lewis BTech PhD MBCS is Lecturer in Medical Computing in the Department of Medical Computing and Statistics, University of Wales College of Medicine and $M$ Charny MA MB BChir PhD MFCM is District Medical Advisor to Bath District Health Authority and formerly was a Lecturer in the Department of Epidemiology and Community Medicine, UWCM.

\section{References}

(1) Harris, J. QALYfying the value of life. Fournal of medical ethics 1987, 13: 117-123.

(2) Jones-Lee M M. The value of life: an economic analysis. London: Martin Robertson, 1976.

(3) Misham E J. Cost-benefit analysis. London: George Allen and Unwin, 1982.

(4) Fuchs V R. Who shall live? USA: Basic Books, 1983.

(5) Charny M, Farrow S, Lewis P A. Who is using cervical cancer screening services. Health trends 1987; 19, 4:3-5.

(6) Charny M, Lewis P A. Choosing who shall not be treated in the NHS. (In preparation).

(7) Smith R. The wasted opportunity of the election. British medical journal 1987; 294: 1438-1439. 